\title{
Optimal Design of Optical Filter for White Light Interferometry based on Sampling Theory
}

\author{
Hidemitsu Ogawa \\ School of Education, Tokyo University of Social Welfare \\ 2020-1, Sanno-cho, Isesaki, Gunma 372-0831, Japan \\ hidemitsu-ogawa@kuramae.ne.jp \\ Akira Hirabayashi \\ Department of Computer Science and Systems Engineering, \\ Yamaguchi University, \\ 2-16-1, Tokiwadai, Ube, Yamaguchi 755-8611, Japan \\ a-hira@yamaguchi-u.ac.jp
}

\begin{abstract}
Vertical-scanning white light interferometry is a technique of profiling the surface topography of objects such as semiconductors, liquid crystal displays (LCDs), and so on. The profile is given by the "squared-envelope function" of the interference signal, which is called the interferogram. The world's fastest surface profiling algorithm exploits a generalized sampling theorem that directly reconstructs the squared-envelope function from an infinite number of samples of the interferogram. The optical system of the white light interferometer is equipped with an optical filter. So far there were no guidelines for designing the optical filter's characteristics. Field engineers empirically chose some combination of commercial optical filters by checking the corresponding interferograms. The main purpose of this paper is to provide a guideline for designing the optical filter's characteristics. That is, we devise the optimal characteristics of the optical filter in the sense that, with a fixed passband of the optical filter, the second moment of the squared interferogram is minimized. Simulation results confirm the effectiveness of the optimal characteristics.
\end{abstract}

Key words and phrases: White light interferometry, Surface profiling, Optimal characteristic of optical filter, Quadrature sampling, Second-order sampling

The IEEE Transactions on Signal Processing EDICS numbers - 2-SAMP 2000 AMS Mathematics Subject Classification - 94A20, 94A12, 49K30, 78A99, 78M50 


\section{Introduction}

Vertical-scanning white light interferometry is a widely used technique of profiling the surface topography of objects such as semiconductors, liquid crystal displays (LCDs), and so on. It is attractive because of its advantages, including non-contact measurement and an unlimited measurement range in principle $[1,2,3,6,7,9,10]$.

Figure 1 shows a basic setup of an optical system used for surface profiling by white light vertical-scanning interferometry (or merely white light interferometry). It is based on the Michelson interferometer. A beam from a white light source passes through an optical filter, which controls the spectrum of the light. The center wavelength and the bandwidth of the optical filter in Figure 1 are denoted by $\lambda_{c}$ and $2 \lambda_{b}$, respectively. Let $k_{l}$ and $k_{u}$ be angular wavenumbers defined by

$$
k_{l}=\frac{2 \pi}{\lambda_{c}+\lambda_{b}}, \quad k_{u}=\frac{2 \pi}{\lambda_{c}-\lambda_{b}} .
$$

We also define

$$
k_{w}=k_{u}-k_{l}
$$

Let $a(k)$ be the characteristic of the optical filter in terms of an angular wavenumber $k$. The support of $a(k)$ is the interval $k_{l}<k<k_{u}$. That is,

$$
a(k)=0 \quad\left(k \leq k_{l}, k \geq k_{u}\right)
$$

Equation (3) means that the parameters $k_{l}$ and $k_{u}$ denote the so-called cutoff frequency of the optical filter in terms of the angular wavenumber. The parameter $k_{w}$ stands for the bandwidth of the optical filter in terms of the angular wavenumber.

A halogen lamp is used as a white light source. It has a limited bandwidth in practice. However, it is very wide compared with the spectral width of the optical filter. For example, a light source has a spectrum from $400 \mathrm{~nm}$ to $800 \mathrm{~nm}$, while the bandwidth of the optical filter is $2 \lambda_{b}=60 \mathrm{~nm}$. Hence, we can assume that the spectrum for light source within the bandwidth of the optical filter is flat.

The lens to the left of the filter serves to parallelize the light from the source. The light beam is reflected by the beam splitter $\mathrm{A}$, and divided into two portions by the beam splitter $\mathrm{B}$ at point $O$. One of the portions indicated by the dotted line is transmitted to a reference mirror at distance $L$ from point $O$. The other portion indicated by the dashed line is transmitted to the surface of an object being observed. Let $z_{p}$ be the height of the surface from the stage at point $P$. The virtual plane at distance $L$ from point $O$ is denoted by $E$. Let $z$ be the distance between the plane $E$ and the stage. 


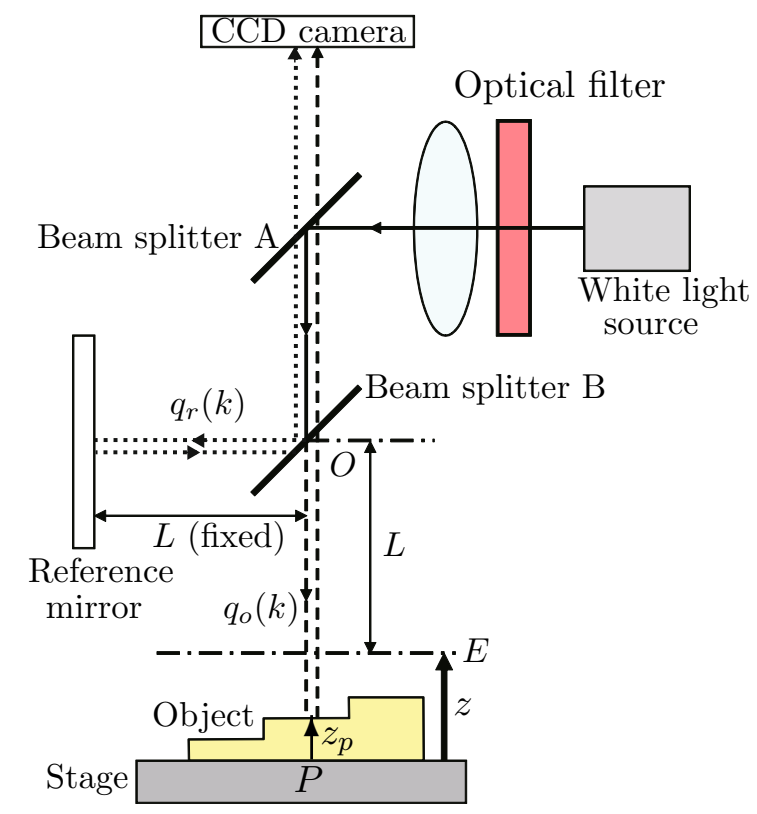

Figure 1: Basic setup of an optical system for surface profiling by verticalscanning white light interferometry.

The two beams reflected by the object surface and the reference mirror are recombined and interfere. As the interferometer moves along the $z$-axis, the resultant beam intensity varies like the dotted line in Figure 2. The curve is called a white light interferogram or simply an interferogram, and denoted by $g(z)$. It is expressed by the sum of the oscillating part $f(z)$ and a constant $C$, as

$$
g(z)=f(z)+C
$$

The function $f(z)$ is also called an interferogram.

Let $q_{o}(k)$ and $q_{r}(k)$ be the averaged attenuation rates of the two beams along the dashed and dotted lines in Figure 1, respectively. Let $\psi(k)$ be

$$
\psi(k)= \begin{cases}2\{a(k)\}^{2} q_{o}(k) q_{r}(k) & (k>0), \\ 0 & (k \leq 0)\end{cases}
$$

The function $\psi(k)$ is also supported on the same interval as $a(k)$. That is,

$$
\psi(k)=0 \quad\left(k \leq k_{l}, k \geq k_{u}\right)
$$

The functions $\psi(k)$ and $a(k)$ are related to the interferogram $f(z)$ and the con- 


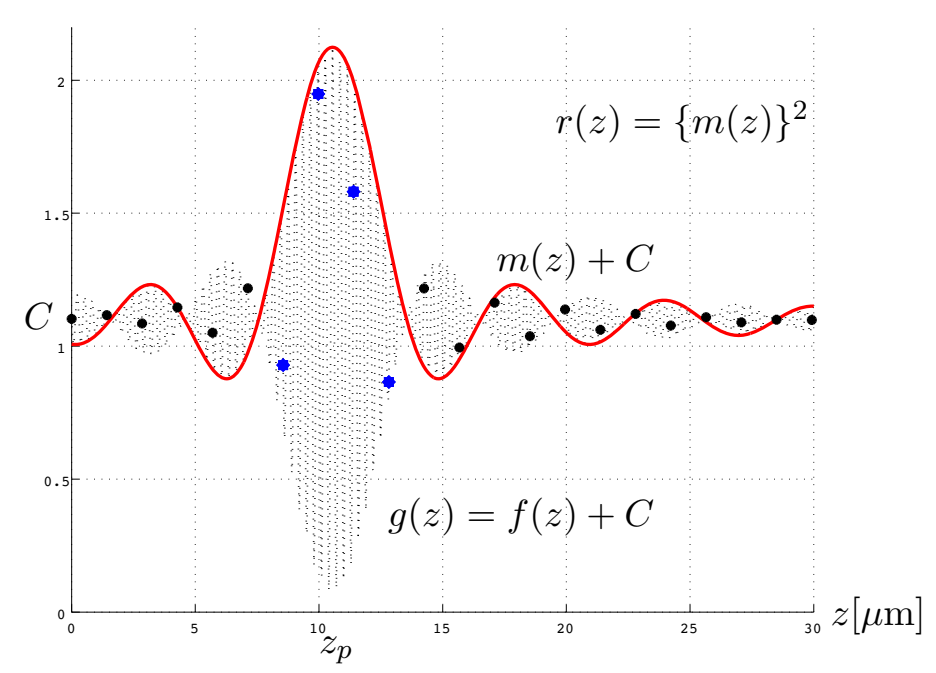

Figure 2: An example of a simulated white light interferogram $g(z)$ (the dotted line), its envelope function (the red thick line), and sampled values of the interferogram in the main lobe (the blue dots) and the side lobes (the black dots).

stant $C$ as

$$
\begin{aligned}
f(z) & =\int_{k_{l}}^{k_{u}} \psi(k) \cos 2 k\left(z-z_{p}\right) d k, \\
C & =\int_{k_{l}}^{k_{u}}\{a(k)\}^{2}\left(\left\{q_{o}(k)\right\}^{2}+\left\{q_{r}(k)\right\}^{2}\right) d k .
\end{aligned}
$$

Equation (7) physically means that $\psi(k)$ is the amplitude of sinusoidal fluctuating part in the light intensity of wavenumber $k$ observed by the CCD detector.

It is important that if the height $z_{p}$ is high, the peak of the interferogram appears in the right side of Figure 2, while if $z_{p}$ is low, it appears in the left side. Hence, the maximum position of the interferogram provides the height $z_{p}$. Let $f_{s}(z)$ be the Hilbert transform of $f(z)$ :

$$
f_{s}(z)=\int_{-\infty}^{\infty} \frac{f\left(z^{\prime}\right)}{z-z^{\prime}} d z^{\prime} .
$$

Then, the envelope function $m(z)$ of $f(z)$ is defined by

$$
m(z)=\sqrt{\{f(z)\}^{2}+\left\{f_{s}(z)\right\}^{2}} .
$$

This is shown by the solid line in Figure 2 and has the same peak as the interferogram as well as its square $r(z)=\{m(z)\}^{2}$. The function $r(z)$ is called the squared-envelope function. These functions, $m(z)$ and $r(z)$, are much smoother than the interferogram. Hence, they can be used for detecting the peak instead of the interferogram. In this paper, we use the latter $r(z)$. 
The intensity of the interferogram is observed by a charge-coupled device (CCD) video camera in Figure 1. It has, for example, $512 \times 480$ detectors. Each detector corresponds to a point on the surface to be measured. All detectors observe intensity of interference light at the same time.

Since the CCD camera has a shutter speed of 1/1000 second and outputs the intensity, for example, every $1 / 60$ second, we can utilize only discrete sampled values of the interferogram shown by ' $\bullet$ ' in Figure 2 . Therefore, if the camera moves at $30 \mu \mathrm{m} / \mathrm{s}$, then the sampling interval yields $0.5 \mu \mathrm{m}$. We have to estimate the maximum position of the interferogram from these sampled values. Here, sampling theory essentially plays the central role.

So far there are no guidelines for designing the optical filter's characteristics. Field engineers empirically chose some combination of commercial optical filters by checking the corresponding interferograms. The main purpose of this paper is to provide a guideline for designing the optical filter's characteristics.

To provide a guideline for designing the optical filter's characteristics, we devise an optimal characteristic of the optical filter in the sense that, with a fixed passband of the optical filter, the second moment of the square of the interferogram is minimized. We show that the optical characteristic is given by a sine curve, which has a half of the period as the fixed passband. We also show the so-called uncertainty relation between the band-width and the second moment of the square of the interferogram.

This paper is organized as follows. Section 2 briefly reviews sampling theorem for squared-envelope functions. In Section 3, we devise the optimal characteristics of the optical filter, the main contribution of the present paper. Three corollaries, including the uncertainty relation, are also shown here. Section 4 is devoted to the proof of the main result given in Section 3. Section 5 provides simulation results that confirm the effectiveness of the optimal characteristics of the optical filter.

\section{Sampling theorem for squared-envelope functions}

From the viewpoint of sampling theory, vertical-scanning white light interferometry has the following two interesting features. First, the signal to be processed, a white light interferogram, $f(z)$, is a bandpass signal. This property comes from (6) and (7). That is, $f(z)$ is a bandpass signal whose Fourier transform is supported in the interval $\left[-2 k_{u},-2 k_{l}\right] \cup\left[2 k_{l}, 2 k_{u}\right]$. Hence, $f(z)$ can be reconstructed from its samples by using the sampling theorem for bandpass signals [8].

Second, the signal to be reconstructed from the sampled values of $f(z)$ is not the interferogram itself, but its squared-envelope function $r(z)$. This type of sampling theorem is called a generalized sampling theorem [4, 11, 12]. Since the squared-envelope function $r(z)$ is the sum of squares of $f(z)$ and its Hilbert 
transform, the squared-envelope function is also reconstructed from samples of $f(z)$, not those of $r(z)$. Indeed, the following result was established $[6,10]$.

Proposition 1 [6] (Sampling theorem for squared-envelope functions) Let I be an integer such that

$$
0 \leq I \leq \frac{k_{l}}{k_{w}},
$$

and $k_{b}$ be any real number that satisfies

$$
\begin{cases}k_{u} \leq k_{b} & (I=0) \\ \frac{k_{u}}{I+1} \leq k_{b} \leq \frac{k_{l}}{I} & (I \neq 0)\end{cases}
$$

Let $\Delta$ be a sampling interval given by

$$
\Delta=\frac{\pi}{2 k_{b}},
$$

and $\left\{z_{n}\right\}_{n=-\infty}^{\infty}$ be sample points defined by

$$
z_{n}=n \Delta .
$$

Then, it holds that

1. When $z$ is a sample point $z_{j}$,

$$
r\left(z_{j}\right)=\left\{f\left(z_{j}\right)\right\}^{2}+\frac{4}{\pi^{2}}\left\{\sum_{n=-\infty}^{\infty} \frac{f\left(z_{j+2 n+1}\right)}{2 n+1}\right\}^{2} .
$$

2. When $z$ is not any sample point,

$$
\begin{aligned}
r(z) & =\frac{2 \Delta^{2}}{\pi^{2}}\left[\left(1-\cos \frac{\pi z}{\Delta}\right)\left\{\sum_{n=-\infty}^{\infty} \frac{f\left(z_{2 n}\right)}{z-z_{2 n}}\right\}^{2}\right. \\
& \left.+\left(1+\cos \frac{\pi z}{\Delta}\right)\left\{\sum_{n=-\infty}^{\infty} \frac{f\left(z_{2 n+1}\right)}{z-z_{2 n+1}}\right\}^{2}\right] .
\end{aligned}
$$

Based on the proposition, the world's fastest surface profiling algorithm was proposed for vertical-scanning white light interferometry and installed in commercial systems [6].

Yet we have the following difficulties when applying Proposition 1 for surface profiling. In Proposition 1, an infinite number of sampled values $\left\{f\left(z_{n}\right)\right\}_{n=-\infty}^{\infty}$ of the interferogram $f(z)$ are used. In practical applications, however, only a 
finite number of sampled values $\left\{g\left(z_{n}\right)\right\}_{n=0}^{N-1}$ of the interferogram $g(z)=f(z)+C$ are available. Hence, we have to truncate the infinite series in Proposition 1 and approximate the sampled values $f\left(z_{n}\right)$ by $g\left(z_{n}\right)-\hat{C}$, where $\hat{C}$ is an estimate of $C$. For example, the average of $g\left(z_{n}\right)$ is used as $\hat{C}$. That is,

$$
\hat{C}=\frac{1}{N} \sum_{n=0}^{N-1} g\left(z_{n}\right) .
$$

Then, the truncation error and the estimation error for $C$ occur. Both of these errors severely affect our final goal of the precise estimation of $z_{p}$.

\section{Optimal characteristics of optical filter}

To reduce both of the errors, the following observation is crucial. As you can see in Figure 2, only several samples are located in the main lobe of $g(z)$ while the rest of them are in side lobes. The samples in the side lobes mostly vanishes once the constant $C$ is precisely estimated. This implies that the smaller the side lobes are, the smaller the truncation error will be. Smaller side lobes also lead us to a better estimation of $C$, as is experimentally shown in Section 5 .

Even though the parameters $k_{l}$ and $k_{u}$ are fixed in this paper, there still exists a freedom in the shape of $a(k)$ on the interval $\left(k_{l}, k_{u}\right)$. Further, $(5)$ and (7) show that we can control the shape of $f(z)$ by the characteristic $a(k)$ of the optical filter through $\psi(k)$. Hence, we can easily arrive at the following idea.

Let us consider the functional of $\psi$ defined by

$$
J[\psi]=\int_{-\infty}^{\infty}\left(z-z_{p}\right)^{2}\{f(z)\}^{2} d z .
$$

We design $\psi(k)$ so that it minimizes the functional $J[\psi]$. We now show the main result in this paper.

Theorem 1 Consider the set of functions $\psi(k)$, which are differentiable on $\left(k_{l}, k_{u}\right)$ and satisfy

$$
\begin{cases}\psi(k)=0 \quad & \left(k \leq k_{l}, k \geq k_{u}\right) \\ \psi(k) \geq 0 & \left(k_{l}<k<k_{u}\right)\end{cases}
$$

and

$$
\int_{k_{l}}^{k_{u}}\{\psi(k)\}^{2} d k=1 .
$$

It holds that

$$
J[\psi] \geq\left(\frac{\pi}{2}\right)^{3} \frac{1}{k_{w}^{2}}
$$




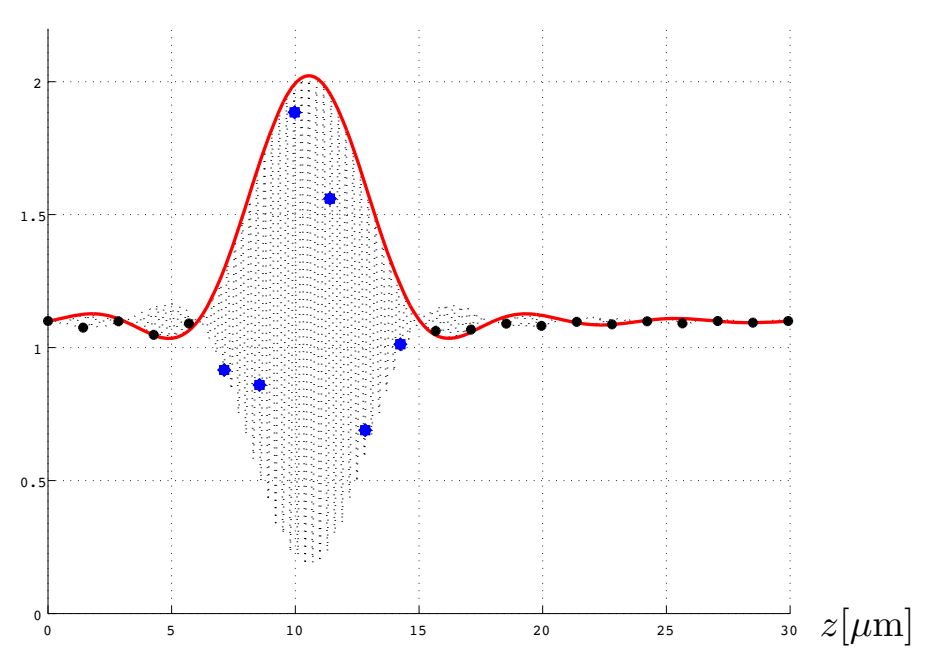

Figure 3: A white light interferogram $g(z)$ for the optimal optical filter (the dotted line), its envelope function (the red thick line), and sampled values of the interferogram. The blue larger and black smaller dots show samples in the main and side lobes, respectively.

with equality if and only if

$$
\psi(k)=\sqrt{\frac{2}{k_{w}}} \sin \frac{\pi\left(k-k_{l}\right)}{k_{w}} \quad\left(k_{l} \leq k \leq k_{u}\right) .
$$

A proof of this theorem is reserved for the next section. The following corollary is a direct consequence from (19) and (5).

Corollary 1 The optimal characteristic a $(k)$ that minimizes $J[\psi]$ in (15) is given by

$$
a(k)=\left(\frac{\sin \left(\pi\left(k-k_{l}\right) / k_{w}\right)}{\sqrt{2 k_{w}} q_{o}(k) q_{r}(k)}\right)^{1 / 2} \quad\left(k_{l} \leq k \leq k_{u}\right) .
$$

Substituting (19) into (7) yields the following corollary.

Corollary 2 The optimal shape of the interferogram $f(z)$ is given by

$$
f(z)=m(z) \cos \left\{\left(k_{u}+k_{l}\right)\left(z-z_{p}\right)\right\},
$$

where

$$
m(z)=\frac{2 \pi \sqrt{2 k_{w}} \cos \left\{k_{w}\left(z-z_{p}\right)\right\}}{\pi^{2}-4 k_{w}^{2}\left(z-z_{p}\right)^{2}} .
$$

The interferogram for the optimal optical filter given by (21) and (22) is shown in Figure 3. The interferogram in Figure 2 was constructed from a rectangular $\psi(k)$ given by

$$
\psi(k)= \begin{cases}1 / \sqrt{k_{w}} & \left(k_{l}<k<k_{u}\right), \\ 0 & \text { (otherwise) }\end{cases}
$$


In both figures, $\lambda_{c}=600 \mathrm{~nm}$ and $\lambda_{b}=30 \mathrm{~nm}$ were used. The sampling interval used in both figures is $\Delta=1.425 \mu \mathrm{m}$, which is the maximum among those satisfying $(9) \sim(11)$. We can see that the side lobes in Figure 3 are much smaller than those in Figure 2, which meets our expectations. On the other hand, the main lobe in Figure 3 is wider than that in Figure 2. In fact, we have six samples in the main lobe in Figure 3 while only four samples are located there in Figure 2. This desirable effect comes from constraint (17) together with the minimization criterion (15). These phenomena increase the accuracy of the estimation $z_{p}$, which will be demonstrated in Section 5 through computer simulations.

Let $\sigma^{2}$ be the second moment of the square of the interferogram defined by (15). It follows from (18) that

Corollary 3 The following uncertainty relation holds.

$$
\sigma^{2} k_{w}^{2} \geq\left(\frac{\pi}{2}\right)^{3}
$$

The equality in this inequality is established by the optimal optical filter.

\section{Proof of Theorem 1}

For the proof we use the following Wirtinger's inequality.

Lemma $1\left[5\right.$, p. 185] Let $\phi$ be any real valued function in $L^{2}$. If $\phi(0)=\phi(\pi)=0$ and $\phi^{\prime}$ is $L^{2}$, then

$$
\int_{0}^{\pi}\{\phi(x)\}^{2} d x \leq \int_{0}^{\pi}\left\{\phi^{\prime}(x)\right\}^{2} d x
$$

with equality if and only if $\phi(x)=c \sin x$ for any real constant $c$.

We shall prove Theorem 1. Let $\omega=2\left(z-z_{p}\right)$ and $F(\omega)=f(z)$. Exactly speaking, $F(\omega)=f\left(\frac{\omega}{2}+z_{p}\right)$ and $f(z)=F\left(2\left(z-z_{p}\right)\right)$. We shall simply denote these relations by $F(\omega)=f(z)$. It follows from (7) that $f\left(2 z_{p}-z\right)=f(z)$. That is, $f(z)$ is an even function with respect to the point $z=z_{p}$. Then we have

$$
F(-\omega)=F(\omega)
$$

That is, $F(\omega)$ is an even function with respect to the origin $\omega=0$.

It follows from (23) and (7) that

$$
\begin{aligned}
2 F(\omega) & =F(\omega)+F(-\omega) \\
& =\int_{k_{l}}^{k_{u}}\{\psi(k)+\psi(-k)\} \cos \omega k d k \\
& =\int_{-\infty}^{\infty}\{\psi(k)+\psi(-k)\} e^{-i \omega k} d k,
\end{aligned}
$$


and hence

$$
F(\omega)=\frac{1}{2} \int_{-\infty}^{\infty}\{\psi(k)+\psi(-k)\} e^{-i \omega k} d k
$$

Then,

$$
i \omega F(\omega)=\frac{1}{2} \int_{-\infty}^{\infty}\left\{\psi^{\prime}(k)-\psi^{\prime}(-k)\right\} e^{-i \omega k} d k .
$$

Since (6) gives $\psi^{\prime}(k) \psi^{\prime}(-k)=0$, applying Parseval's formula to (25) and considering (6) yield

$$
\frac{1}{2 \pi} \int_{-\infty}^{\infty}\{\omega F(\omega)\}^{2} d \omega=\frac{1}{2} \int_{-\infty}^{\infty}\left\{\psi^{\prime}(k)\right\}^{2} d k=\frac{1}{2} \int_{k_{l}}^{k_{u}}\left\{\psi^{\prime}(k)\right\}^{2} d k .
$$

It follows from (15) and (26) that

$$
\begin{aligned}
J[\psi] & =\int_{-\infty}^{\infty}\left(z-z_{p}\right)^{2}\{f(z)\}^{2} d z \\
& =\frac{\pi}{4} \frac{1}{2 \pi} \int_{-\infty}^{\infty}\{\omega F(\omega)\}^{2} d \omega \\
& =\frac{\pi}{8} \int_{k_{l}}^{k_{u}}\left\{\psi^{\prime}(k)\right\}^{2} d k .
\end{aligned}
$$

In order to use Lemma 1, let

$$
k^{\prime}=\frac{\pi}{k_{w}}\left(k-k_{l}\right)
$$

and $\phi\left(k^{\prime}\right)=\psi(k)$. Equation (16) yields $\phi(0)=\phi(\pi)=0$ and $\phi^{\prime} \in L^{2}$. It follows from $(27),(17)$ and Lemma 1 that

$$
\begin{aligned}
J[\psi] & =\frac{\pi}{8} \int_{k_{l}}^{k_{u}}\left\{\psi^{\prime}(k)\right\}^{2} d k \\
& =\frac{\pi}{8} \frac{\pi}{k_{w}} \int_{0}^{\pi}\left\{\phi^{\prime}\left(k^{\prime}\right)\right\}^{2} d k^{\prime} \\
& \geq \frac{\pi}{8} \frac{\pi}{k_{w}} \int_{0}^{\pi}\left\{\phi\left(k^{\prime}\right)\right\}^{2} d k^{\prime} \\
& =\frac{\pi}{8}\left(\frac{\pi}{k_{w}}\right)^{2} \int_{k_{l}}^{k_{u}}\{\psi(k)\}^{2} d k \\
& =\left(\frac{\pi}{2}\right)^{3} \frac{1}{k_{w}^{2}} .
\end{aligned}
$$




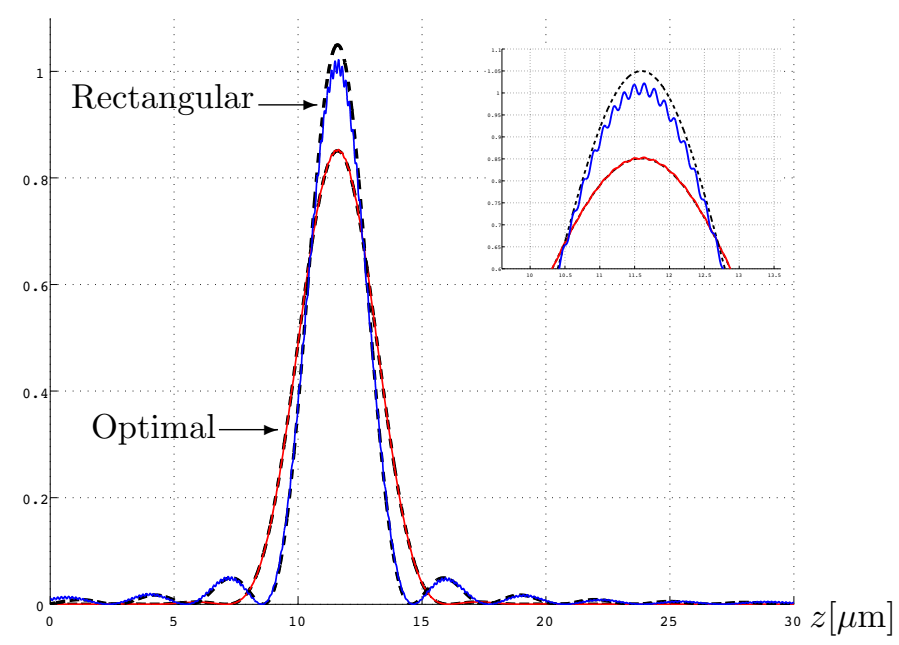

Figure 4: Squared-envelope functions (dashed lines) and reconstructed functions (solid lines) from samples of $g(z)$ for both the optimal and the rectangular $\psi(k)$. The peak portions of the curves were magnified in the small top-right box.

Hence we have (18). From Lemma 1 the equality in (18) holds if and only if $\phi\left(k^{\prime}\right)=c \sin k^{\prime}$ for any real constant $c$. Substituting this $\phi$ into (17) yields

$$
\begin{aligned}
1 & =\int_{k_{l}}^{k_{u}}\{\psi(k)\}^{2} d k \\
& =c^{2} \int_{k_{l}}^{k_{u}}\left(\sin \frac{\pi\left(k-k_{l}\right)}{k_{w}}\right)^{2} d k \\
& =\frac{c^{2} k_{w}}{2} .
\end{aligned}
$$

Then we have

$$
c=\sqrt{\frac{2}{k_{w}}}
$$

because of (16). This implies (19), which completes the proof.

\section{Simulations}

We compared the optimal and rectangular characteristics $\psi(k)$ by computer simulations. We first sampled the interferograms $g(z)$ generated from both functions $\psi(k)$ with the sampling interval $\Delta=1.425 \mu \mathrm{m}$. Then, the averages for each sample values were computed for the estimation of $C$. Finally, we reconstructed the squared-envelope functions $r(z)$ by using a finite number of $g\left(z_{n}\right)-\hat{C}$ instead of 
$f\left(z_{n}\right)$ in Proposition 1. The reconstructed functions are shown in Figure 4 by the solid lines as well as the original squared-envelope functions by the dashed lines for both the optimal and the rectangular $\psi(k)$. The peak portions of the curves were magnified in the small top-right box. We can see that the reconstructed function for the optimal $\psi(k)$ provides a much better result than that for the rectangular $\psi(k)$. We also notice that the latter oscillates severely. This may cause difficulties for a fast search of the maximum position.

We repeated the same simulations for thirty two values of $z_{p}$ from $10 \mu \mathrm{m}$ to $20 \mu \mathrm{m}$. The averages of estimation errors for $z_{p}$ were $0.0496 \mu \mathrm{m}$ and $0.0541 \mu \mathrm{m}$ for the optimal and the rectangular $\psi(k)$, respectively. The averages of normalized truncation errors were $0.35 \%$ and $4.67 \%$ for the optimal and the rectangular $\psi(k)$, respectively. The former is less than $7.5 \%$ of the latter. These results show the effectiveness of the optimal characteristics of the optical filter.

\section{ACKNOWLEDGEMENT}

Grateful thanks are extended to Dr. Katsuichi Kitagawa of Toray Engineering Co., Ltd. for his discussions. The authors are also very thankful to the anonymous reviewers for their valuable comments.

\section{References}

[1] P. J. Caber, Interferometric profiler for rough surfaces, Applied Optics, 32(19), 3438-3441, 1993.

[2] S. S. C. Chim and G. S. Kino, Three-dimensional image realization in interference microscopy, Applied Optics, 31(14), 2550-2553, 1992.

[3] P. de Groot and L. Deck, Surface profiling by analysis of white-light interferograms in the spatial frequency domain, Journal of Modern Optics, 42(2), 389-401, 1995.

[4] O.D. Grace and S.P. Pitt, Sampling and interpolation of bandlimited signals by quadrature methods, Journal of the Acoustical Society of America, 48(6), 1311-1318, 1969.

[5] G.H. Hardy, J. E. Littlewood and G.Polya, Inequalities, 2nd Ed., Cambridge University Press, Cambridge, 1999.

[6] A. Hirabayashi, H. Ogawa, and K. Kitagawa, Fast surface profiler by whitelight interferometry by use of a new algorithm based on sampling theory, Applied Optics, 41(23), 4876-4883, 2002.

[7] G. S. Kino and S. S. C. Chim, Mirau correlation mictroscope. Applied Optics, 29(26), 3775-3783, 1990. 
[8] A. Kohlenberg, Exact interpolation of band-limited functions, Journal of Applied Physics, 24, 1432-1436, 1953.

[9] K. G. Larkin, Efficient nonlinear algorithm for envelope detection in white light interferometry, Journal of Optical Society of America, 13(4), 832-843, 1996.

[10] H. Ogawa and A. Hirabayashi, Sampling theory in white-light interferomtery, Sampl. Theory Signal Image Process., 1(2), 87-116, 2002.

[11] D. W. Rice and K.H. Wu, Quadrature sampling with high dynamic range, IEEE Transactions on Aerospace and Electronic Systems, AES-18(4), 736739, 1982.

[12] W. M. Waters and B.R. Jarrett, Bandpass signal sampling and coherent detection, IEEE Transactions on Aerospace and Electronic Systems, AES18(4), 731-736, 1982. 